\title{
Pneumonia nosocomial: diagnóstico e intervenções de enfermagem
}

\author{
Nosocomial pneumonia: diagnostic and intervention of nursing \\ La neumonía nosocomial: diagnóstico e intervenciones de enfermería
}

Julya Carolline Folle Alves ${ }^{1}$; Kattyelly Cristina Grando ${ }^{2}$; Leo Christyan Alves Lima ${ }^{3}$; Jéssica Mayara Alves Pinto ${ }^{4}$; Maysa Silva Sampaio ${ }^{5}$; Kelmi Cristina Sarancini ${ }^{6}$; Laurindo Pereira Souza $^{7}$

\begin{abstract}
Resumo
A pneumonia é uma doença infecciosa, causada por bactérias, vírus, fungos, parasitas e outros microrganismos, sendo a maioria causada por agentes bacterianos. O objetivo da pesquisa foi buscar dados na literatura sobre o estabelecimento da pneumonia nosocomial e propor os principais os diagnósticos e intervenções de enfermagem. Trata-se de um estudo exploratório qualitativo, descritivo realizada por meio de revisão bibliográfica. Os dados foram coletados em ${ }^{i}$ revistas e periódicos disponíveis na internet, por meio da busca em base de dados: LILACS (Literatura Latino Americano e do Caribe em Ciências da Saúde), SciELO (Scientific Eletronic Library On-line) e BDENF (Banco de Dados em Enfermagem). O período abordado de revisão e seleção dos artigos foi de 2004 a 2014. As pneumonias nosocomiais, são definidas como uma infecção pulmonar adquirida durante a estadia no hospital que ocorrem 48 horas após a internação hospitalar. O diagnóstico de Enfermagem constitui a base para a seleção das intervenções de enfermagem para o alcance dos resultados dos quais o enfermeiro é responsável. Conclui-se que o enfermeiro tem um papel fundamental, frente a um paciente com pneumonia sistematizando as ações de enfermagem.
\end{abstract}

Descritores: Pneumonia nosocomial, Enfermagem, Diagnóstico, Intervenções

\footnotetext{
Abstract

Pneumonia is an infectious disease caused by bacteria, viruses, fungi, parasites and other microorganisms, mostly caused by bacterial agents. The aim was to fetch data in the literature on the establishment of nosocomial pneumonia and propose the main diagnoses and nursing interventions. This is a qualitative exploratory, descriptive study performed by literature review. Data were collected in magazines and periodicals available on the internet by searching in database: LILACS (Latin American and Caribbean Health Sciences), SciELO (Scientific Electronic Library Online) and BDENF (Database Nursing). The period covered the review and selection of the

${ }^{1}$ Graduanda em Enfermagem pela Faculdade de Ciências Biomédicas de Cacoal (FACIMED). email: julya_caroline@hotmail.com

${ }^{2}$ Graduanda em Enfermagem pela Faculdade de Ciências Biomédicas de Cacoal (FACIMED). email: kattygrando@hotmail.com

${ }^{3}$ Graduando em Enfermagem pela Faculdade de Ciências Biomédicas de Cacoal (FACIMED). email: leochristyan@hotmail.com

${ }^{4}$ Graduanda em Enfermagem pela Faculdade de Ciências Biomédicas de Cacoal (FACIMED). email: jessica2013alves@hotmail.com

${ }^{5}$ Graduando em Enfermagem pela Faculdade de Ciências Biomédicas de Cacoal (FACIMED). email: maysasampaio@hotmail.com

${ }^{6}$ Graduanda em Enfermagem pela Faculdade de Ciências Biomédicas de Cacoal (FACIMED). email: kelmi_cris@hotmail.com

${ }^{7}$ Mestre em Ciências da Saúde pelo IAMSPE-SP. Docente da Faculdade de Ciências Biomédicas de Cacoal (FACIMED). email: laurindosorrisox@ hotmail.com
} 103 
ISSN 2179-6750

articles was from 2004 to 2014. Nosocomial pneumonia, are defined as a lung infection acquired during the hospital stay that occur 48 hours after hospitalization. The diagnosis of Nursing is the basis for selection of nursing interventions to achieve the results of which the nurse is responsible. It is concluded that the nurse has a key role, we have a patient with pneumonia systematizing the nursing actions.

Key-words: Nosocomial pneumonia, Nursing, Diagnosis, Interventions

\section{Resumen}

La neumonía es una enfermedad infecciosa causada por bacterias, virus, hongos, parásitos y otros microorganismos, en su mayoría causadas por agentes bacterianos. El objetivo era obtener los datos en la literatura sobre el establecimiento de la neumonía nosocomial y proponer los principales diagnósticos e intervenciones de enfermería. Se trata de un estudio cualitativo exploratorio, descriptivo realizado por revisión de la literatura. Los datos fueron recogidos en revistas y periódicos disponibles en Internet a través de la búsqueda en la base de datos LILACS (Ciencias de América Latina y el Caribe de la Salud), SciELO (Scientific Electronic Library Online) y BDENF (Base de Datos de enfermería). El período cubierto la revisión y selección de artículos fue de 2004 a 2014. La neumonía nosocomial, se define como una infección pulmonar adquirida durante la estancia hospitalaria que se producen 48 horas después de la hospitalización. El diagnóstico de enfermería es la base para la selección de las intervenciones de enfermería para lograr los resultados, que la enfermera es responsable. Se llegó a la conclusión de que la enfermera tiene un papel clave en la parte delantera de un paciente con neumonía sistematizar las acciones de enfermería.

Palabras-claves: Neumonía Nosocomial, Enfermería, Diagnóstico, Intervención

\section{Introdução}

A pneumonia é uma doença infecciosa, causada por bactérias, vírus, fungos, parasitas e outros microrganismos, sendo a maioria causada por agentes bacterianos. Nesta infecção, os bronquíolos respiratórios e alvéolos são preenchidos por exsudato inflamatório dificultando as trocas gasosas. Normalmente a doença se desenvolve quando há uma falha nos mecanismos de defesa do organismo, na qual o interior dos alvéolos fica todo ou parcialmente preenchida por líquido e restos celulares, mudando as funções gasosas em diferentes períodos da doença, progredindo e comprometendo a função pulmonar ${ }^{1}$.

Entre as doenças mais comuns do sistema respiratório inferior, destacam-se as pneumonias. A taxa de internações por pneumonia vem diminuindo nos últimos anos, porém continua elevada. Segundo dados do Sistema de Informações Hospitalares do Sistema Único de Saúde, ocorreram 733.209 internações por pneumonia no Brasil em 2007, correspondendo à primeira causa de internação pela doença. Entre os pacientes internados, 5\% a 10\% são encaminhados para Unidade de Tratamento Intensivo (UTI) ${ }^{2}$. 
De acordo com uma avaliação feita pela Sociedade Brasileira de Pneumologia e Tisiologia ${ }^{3}$, dos microrganismos que possa causar a pneumonia, o $S$. pneumoniae permanece como o mais frequente, seguido pelo $H$. influenzae, Mycoplasma pneumoniae, Chlamydophila pneumoniae, Legionella sp. Outros agentes encontrados em grupos especiais são as enterobactérias, Pseudomonas aeruginosa, S. aureus, anaeróbios e vírus respiratórios (influenza, adenovírus, vírus sincicial respiratório, parainfluenza, coronavírus).

Segundo Silveira ${ }^{19}$, a pneumonia pneumocócica é o tipo mais comum de pneumonia primária, adquirida na comunidade, sendo causa frequente de morbidade e mortalidade, chegando a $80 \%$ dentre todas as pneumonias.

Os fatores de risco independentes associado à pneumonia hospitalar são, desnutrição, doença renal crônica, anemia, consciência deprimida, internação em relação ao mês anterior e cirurgia torácica. Depressão do nível de consciência está associada com uma diminuição da capacidade de proteger as vias respiratórias e podem aumentar o risco de aspiração ${ }^{4}$.

As pneumonias nosocomiais (PN), ou adquiridas no hospital, são definidas como uma infecção pulmonar adquirida durante a estadia no hospital, e representa um imenso peso nos crescentes cursos com a saúde, sem contar os impactos adversos esperados com a doença 5 .

Segundo Morais ${ }^{6}$ a pneumonia nosocomial é a segunda infecção hospitalar mais comum, mas a primeira causa infecciosa em unidades fechadas, responsável por altas taxas de morbidade, mortalidade e aumento expressivo dos custos hospitalares.

O enfermeiro atua de forma sistematizada e individualizada diante dos aspectos clínicos apresentados pelo paciente, buscando a recuperação do estado de saúde do indivíduo frente às suas condições fisiológicas normais ${ }^{7}$.

Conforme Ornelas e Cobucci ${ }^{8}$, o enfermeiro geralmente se depara com pacientes com distúrbios do sistema respiratório, em especial a pneumonia que é uma doença inflamatória que atinge os pulmões e possui diversas causas, para isso, torna-se importante que o mesmo esteja devidamente preparado para programar o plano terapêutico de cuidados sob o auxílio dos diagnósticos de enfermagem que contribuem na escolha dos cuidados de enfermagem, para que haja o alcance dos resultados pelo qual é responsável. Tendo em vista que a pneumonia é uma patologia que atinge comumente as pessoas, notou-se que as literaturas de enfermagem que abordam os diagnósticos de enfermagem relacionados a essa patologia são escassas uma vez que, é de extrema importância o levantamento desses diagnósticos, pois permite agilidade e facilidade nas escolhas 
ISSN 2179-6750

das intervenções de enfermagem.

Diante do exposto o objetivo deste estudo foi buscar dados na literatura sobre o estabelecimento da pneumonia nosocomial e propor os principais os diagnósticos e intervenções de enfermagem.

\section{Metodologia}

Trata-se de um estudo exploratório qualitativo, descritivo realizada por meio de revisão bibliográfica, com a finalidade de analisar as produções acerca da PN e os principais diagnósticos e intervenções de enfermagem aos pacientes acometidos por esta doença.

A pesquisa bibliográfica tem como objetivo procurar explicar um problema a partir de referências teóricas publicadas em documentos, podendo ser, desenvolvida a partir de material já elaborado, constituído principalmente de manuais, artigos científicos, dissertações de mestrados e teses de doutorado ${ }^{4}$, além de busca no acervo da biblioteca Maria da Penha Lenci Campos da Faculdade de Ciências Biomédicas da Cacoal FACIMED.

A pesquisa exploratória é desenvolvida para proporcionar uma visão ampla de determinados $\operatorname{assuntos}^{10}$. O estudo descritivo identifica, sistematiza e classifica as características dos fenômenos. Por meio desta dinâmica surgem novos estudos descritivos que procuram explicar esses fenômenos, ou seja, analisar as variáveis que influenciam ou causam o surgimento dos fatos ${ }^{9,12}$.

Os dados foram coletados em revistas e periódicos disponíveis na internet, por meio da busca em base de dados: LILACS (Literatura Latino Americano e do Caribe em Ciências da Saúde), SciELO (Scientific Eletronic Library On-line) e BDENF (Banco de Dados em Enfermagem), tendo por descritores Decs: "Pneumonia nosocomial"; "Enfermagem"; "Diagnóstico"; "Intervenções de enfermagem". Foi encontrado cerca de 50 (cinquenta) artigos. Adentrando-se aos critérios de inclusão foram utilizados somente os artigos que tivessem uma aproximação maior ao tema proposto. Já os critérios de exclusão, foram àqueles artigos, que não abordassem relativamente ao tema. Sendo assim, dentre esses artigos foram escolhidos 14 (quatorze) artigos, pois os mesmos enfocavam exatamente o tema proposto, estando, portanto, dentro dos critérios de inclusão, relacionado ao tema proposto publicados em periódicos entre os anos de 2004 a 2014 de modo a torná-lo mais claro para a construção de questões importantes para a pesquisa. 
ISSN 2179-6750

\section{Resultados e Discussão}

Pneumonia Nosocomial

Pneumonias nosocomiais são aquelas que ocorrem 48 horas após a internação hospitalar. O principal fator de risco para o surgimento da pneumonia nosocomial é a ventilação mecânica, levando a uma conceituação própria de pneumonia associada à ventilação mecânica aquela que surge 48-72 horas após intubação endotraqueal e suporte ventilatório invasivo ${ }^{12}$.

Pacientes intubados e submetidos à ventilação mecânica contínua constituem-se em um risco de 6 a 21 vezes maiores em adquirir pneumonia $(\mathrm{PN})$ quando comparados àqueles em ventilação espontânea na ausência de vias aéreas artificiais ${ }^{7}$. Ainda segundo Dandagi ${ }^{13}$, a mortalidade em consequência da Pneumonia Associada à ventilação Mecânica (PAVM) é especialmente alta quando é causada por organismos multirresistentes a drogas como os pseudômonas ou espécies de Acinetobacter.

Os pacientes que necessitam de intubação e ventilação mecânica correm risco particular, assim como aqueles com comprometimento da função imune, doenças pulmonares crônicas e instrumentação das vias respiratórias, como intubação endotraqueal ou traqueotomia ${ }^{14}$.

Segundo Rodrigues ${ }^{15}$, a pneumonia nosocomial, é a infecção Associada à Assistência de Saúde (IRAS) que é diagnosticada após 48 horas da internação e que não estava em incubação no momento da chegada ao hospital. É classificada ainda, como pneumonia hospitalar (PH), pneumonia associada à ventilação mecânica (PAV) e pneumonia associada à assistência de saúde (PRAS). As pneumonias hospitalares (PH) são a segunda causa mais freqüente de IRAS, correspondem a aproximadamente a um quarto de todas as Infecções Hospitalares (IHs) em Unidades de Terapia Intensiva (UTI). A PH é aquela que ocorre após 48h da admissão hospitalar, geralmente tratada na unidade de internação (enfermaria/apartamento), não se relacionando à intubação endotraqueal e VM (ventilação mecânica); podendo, entretanto, ser encaminhada para tratamento em UTI, quando se apresenta ou evolui de forma grave.

Para Rodrigues ${ }^{15}$ as implicações etiológicas, terapêuticas e prognósticas, da $\mathrm{PH}$, tem sido classificada quanto ao tempo decorrido desde a admissão até o seu aparecimento. A pneumonia precoce é a que ocorre até o quarto dia de internação, sendo considerada tardia a que se inicia após cinco dias da hospitalização. Quando a PH acomete pacientes em ventilação mecânica são denominadas de Pneumonias Associadas à Ventilação Mecânica (PAVM).

A incidência de PN varia de hospital para hospital. A incidência de PN nas UTIs varia de 9

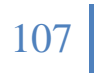


ISSN 2179-6750

a 24\%, com variação relativa aos cuidados apresentados na UTI e diferenças no diagnóstico e nas técnicas usadas ${ }^{13}$.

De acordo com Rufino et al ${ }^{16}$ em geral, entre 0,4 a $1,1 \%$ dos pacientes internados desenvolvem pneumonia, sendo esta a segunda causa infecciosa em unidades fechadas. Apesar da baixa incidência, há elevada mortalidade, de 20 a 50\%, especialmente quando associada a outras doenças e a agentes microbianos multirresistentes.

Segundo Dandagi ${ }^{13}$, atualmente, o papel exato da infecção nosocomial na piora do prognóstico de pacientes de UTI é difícil avaliar, já que tais pacientes estão em estado grave e, portanto, seu estado clínico é grave o suficiente para exigir atendimento na UTI e potencialmente causar a morte.

As infecções nosocomiais são comuns em pessoas hospitalizadas com grave doença de base, imunossupressão ou com prolongadas terapias com antibióticos. Aqueles que necessitam de ventilação mecânica representam um grupo de alto risco, e as infecções adquiridas nessas situações recebem uma designação distinta, pneumonia associada ao ventilador 5 .

Segundo Ricci ${ }^{17}$, pacientes com idade entre 50 a 59 anos são os que mais apresentam pneumonia nosocomial, correspondendo a 31,82\% dos indivíduos, enquanto os pacientes com idade entre 0 a 9 anos e os indivíduos de 10 a 19 anos de idade são os que menos desenvolvem esta patologia, representando $4,55 \%$ dos pacientes.

O diagnóstico da pneumonia hospitalar inclui a progressão ou o surgimento de novo infiltrado pulmonar, febre, leucocitose, secreção brônquica purulenta ${ }^{7}$.

Freire et al ${ }^{18}$, identificaram que as medidas de prevenção em especial as pneumonias são essenciais, devendo ser implantadas estratégias de controle centrando suas ações na padronização e no treinamento de condutas para a assistência aos pacientes de risco. De maneira geral, o sucesso depende do envolvimento de toda a equipe de atendimento, sendo fundamental a educação continuada de todos os profissionais que trabalham com pacientes predispostos a esta doença.

\section{A Enfermagem frente à pacientes com pneumonia}

Conhecer e compreender os diagnósticos de enfermagem que podem estar presentes nos pacientes com quadros de pneumonia é de fundamental importância ao enfermeiro, o que poderá possibilitar agilidade e facilidade nas escolhas das intervenções de enfermagem que o favorecerá no alcance dos resultados pelos quais é responsável ${ }^{8}$. 
ISSN 2179-6750

Entender o diagnóstico e o tratamento da pneumonia é necessário o entendimento dos princípios fisiológicos da respiração e das trocas gasosas, uma vez que, algumas dessas patologias podem originar-se de ventilação inadequada, anormalidades na difusão através da membrana pulmonar e do transporte de gases dos pulmões para os tecidos ${ }^{1}$.

O nível de gravidade da pneumonia define condutas como internação, investigação mais ampla e tratamento mais abrangente. Sendo assim, é necessário caracterizar essa gravidade que pode ser real, existente no momento do diagnóstico, ou potencial ${ }^{19}$.

Segundo a Nanda International ${ }^{20}$, o diagnóstico de enfermagem constitui a base para a seleção das intervenções de enfermagem para o alcance dos resultados dos quais o enfermeiro é responsável.

O diagnóstico de enfermagem (DE) é parte integrante do processo de trabalho deste profissional, permitindo ao mesmo que identifique os problemas, subsidiando a prescrição dos cuidados $^{21}$. Ele auxilia o enfermeiro a elaborar e executar um plano de ação para que assim ele se aproxime de seu objeto de trabalho através de ações programadas, baseando-se nos problemas detectados no paciente. Com isso a produtividade reflete a melhora no processo de trabalho através da qualidade das ações ${ }^{22}$.

Assim como relata Rezende ${ }^{22}$, o histórico de enfermagem é fundamental para a detecção da pneumonia. É importante identificar a gravidade, a localização e a causa da dor torácica. A enfermeira deve monitorizar as alterações na temperatura e no pulso; quantidade odor e coloração das secreções; frequência e gravidade da tosse; grau de taquipneia ou dispneia; alterações nos achados do exame físico (basicamente os encontrados pela inspeção e ausculta do tórax).

Conforme Dandagi ${ }^{13}$, os critérios diagnósticos clínicos de PN e PAVM baseia-se na suspeita clínica de pneumonia com um novo ou progressivo infiltrado radiográfico após 48 horas de internação ou após 48 horas de pacientes sob ventilação mecânica, febre $>38,3^{\circ}$, leucocitose > 12000/cmm ou leucopenia < 4000/cmm, secreções respiratórias purulenta com coloração de Gram demonstração de bactérias e polimorfos, culturas com crescimento > 106 unidades formadoras de colônias/mL (UFC).

O diagnóstico clínico tradicional das pneumonias baseia-se nos dados de anamnese e na presença de estertores crepitantes, entretanto, na criança, taquipneia é considerado o melhor meio de identificar infecção do trato respiratório inferior. Segundo normas da Organização Mundial da Saúde (OMS), a presença de taquipneia e tiragem intercostal e subcostal são suficientes para o 
ISSN 2179-6750

diagnóstico clínico de pneumonia em atendimento primário ${ }^{7}$.

A febre e os calafrios ou a sudorese noturna, em qualquer paciente, associados a quaisquer sintomas respiratórios, devem alertar a enfermeira quanto à possibilidade de pneumonia bacteriana. O exame respiratório, posteriormente identificará as manifestações clínicas da pneumonia: dor tipo pleurítica, fadiga, taquipneia, uso de músculos acessórios da respiração, bradicardia ou relativa bradicardia, tosse e escarro purulento ${ }^{23}$.

Segundo Goldman \& Dennis ${ }^{24}$, na prática, o diagnóstico deve basear-se nas manifestações clínicas sugestivas e radiografias de tórax, com ou sem dados microbiológicos corroborativos. Como o Streptococcus pneumoniae é o agente etiológico mais comum da pneumonia comunitária, praticamente todos os regimes terapêuticos recomendados devem fornecer cobertura adequada para esse patógeno.

Em UTIs para definir a resolução clínica, podemos avaliar leucocitose, temperatura corporal, secreções purulenta, hipoxemia (como $\left.\mathrm{PaO}_{2} / \mathrm{FiO}_{2}\right)$ e achados radiológicos. Embora a temperatura continue a ser um marcador precoce de resolução em tais pacientes, a sua percentagem de anomalias de leucócitos e de oxigenação permanece elevado após a primeira semana de tratamento, enquanto eliminação de secreções e resoluções radiológicas são considerados achados $\operatorname{atrasados}^{25}$.

O tratamento atual de PN depende de adequada terapia antimicrobiana e antibioticoterapia inadequada está associado a maiores taxas de mortalidade ${ }^{13}$.

Segundo Sopena et al ${ }^{29}$, a PN em desenvolvimento em enfermarias geralmente tem um início atrasado e é mais frequente em enfermarias de clínica médica e em pacientes com comorbidades graves. O conhecimento dos fatores de risco para a PH deve melhorar a fiscalização nesse grupo de pacientes para permitir cedo o diagnóstico e tratamento da $\mathrm{PH}$ e melhorar o prognóstico. Além disso, a prevenção de PN em enfermarias gerais devem depender de intervenções, modificar fatores de riscos, tais como desnutrição, anemia e risco de aspiração. Finalmente, a PN fora da UTI carrega elevada morbidade e mortalidade e aumenta o comprimento de internação e da taxa de descarga para uma instalação de enfermagem especializada.

São inúmeros os cuidados que os enfermeiros devem ter com os pacientes, no que diz respeito ao tratamento da pneumonia. Como estão em contato direto com os doentes, é imprescindível que eles tomem medidas que visem à melhora destes.

O quadro 1 abaixo mostra os principais diagnósticos de enfermagem, suas metas e suas 110 


\author{
ISSN 2179-6750
}

respectivas intervenções.

Quadro 1. Cuidados de enfermagem ao paciente com pneumonia: diagnóstico de enfermagem, metas e intervenções de enfermagem

\begin{tabular}{|c|c|c|}
\hline $\begin{array}{l}\text { Diagnóstico de } \\
\text { Enfermagem }\end{array}$ & Metas & Intervenção de Enfermagem \\
\hline $\begin{array}{l}\text { Troca de gases prejudicada } \\
\text { relacionada pela lesão } \\
\text { membrana alvéolo capilar, } \\
\text { evidenciado por dispnéia, } \\
\text { confusão, respiração anormal. }\end{array}$ & $\begin{array}{l}\text { Participar nas atividades } \\
\text { para maximizar a } \\
\text { oxigenação e a limpeza das } \\
\text { vias aéreas. }\end{array}$ & $\begin{array}{l}\text { Aspiração de vias aéreas; Assistência } \\
\text { ventilatória; Controle ácido-base; } \\
\text { Controle de vias aéreas; Estimulo à } \\
\text { tosse; Monitorização respiratória; } \\
\text { Oxigenoterapia; Promoção do } \\
\text { exercício; Terapia com exercícios: } \\
\text { Deambulação. }\end{array}$ \\
\hline $\begin{array}{l}\text { Dor aguda relacionada à } \\
\text { experiência sensorial e } \\
\text { emocional desagradável } \\
\text { evidenciado por dor torácica } \\
\text { (pleurítica), agravada pela } \\
\text { tosse; cefaléia; mialgia; } \\
\text { artralgia. }\end{array}$ & $\begin{array}{l}\text { Controlar a dor mantendo o } \\
\text { conforto ao paciente. }\end{array}$ & $\begin{array}{l}\text { Administração de medicamentos; } \\
\text { Assistência à Analgesia Controlada } \\
\text { pelo Paciente; Controle da dor; } \\
\text { Redução da ansiedade; Suporte } \\
\text { emocional; Terapia com exercícios: } \\
\text { controle muscular, deambulação, } \\
\text { equilíbrio, mobilidade articular. }\end{array}$ \\
\hline $\begin{array}{l}\text { Padrão respiratório ineficaz } \\
\text { relacionado à limitação do } \\
\text { fluxo de ar, evidenciado por } \\
\text { dispneia, tosse e presença de } \\
\text { secreções. }\end{array}$ & $\begin{array}{l}\text { Melhorar o padrão } \\
\text { respiratório; diminuir a } \\
\text { presença de secreções } \\
\text { brônquicas em } 48 \text { horas. }\end{array}$ & $\begin{array}{l}\text { Monitorar o estado respiratório } \\
\text { (frequência respiratória, uso da } \\
\text { musculatura acessória, retrações e } \\
\text { oscilação das narinas, cianose, sibilos } \\
\text { e tosse); Oferecer oxigenoterapia } \\
\text { conforme a prescrição médica e se } \\
\text { necessário; Manter cabeceira da cama } \\
\text { elevada a } 45^{\circ} \text {; Aspiração de vias } \\
\text { aéreas; Assistência ventilatória. }\end{array}$ \\
\hline $\begin{array}{l}\text { Termorregulação ineficaz } \\
\text { relacionado à capacidade } \\
\text { diminuída de manter a } \\
\text { temperatura corporal, } \\
\text { evidenciado por elevações de } \\
\text { temperatura acima de } 37^{\circ} \mathrm{C} \text {. }\end{array}$ & $\begin{array}{l}\text { O cliente deverá apresentar } \\
\text { melhora da temperatura } \\
\text { corporal em } 48 \text { horas após o } \\
\text { início do tratamento. }\end{array}$ & $\begin{array}{l}\text { Regulação da temperatura; Verificar } \\
\text { a temperatura axilar de } 4 / 4 \text { horas e } \\
\text { SSVV. Controle hídrico; Realizar } \\
\text { banho ou compressas frias se a } \\
\text { temperatura não ceder com a } \\
\text { medicação prescrita. }\end{array}$ \\
\hline $\begin{array}{l}\text { Intolerância a atividade } \\
\text { relacionado comprometimento } \\
\text { da função pulmonar } \\
\text { evidenciado por dispnéia ao } \\
\text { menor esforço. }\end{array}$ & $\begin{array}{l}\text { Aumentar a tolerância à } \\
\text { atividade recreativa. }\end{array}$ & $\begin{array}{l}\text { Controle de energia; Assistência no } \\
\text { autocuidado: atividades essenciais na } \\
\text { vida diária; Orientar atividades que } \\
\text { não exijam esforço físico demasiado; } \\
\text { Promoção da mecânica corporal; } \\
\text { Promoção do exercício. }\end{array}$ \\
\hline $\begin{array}{l}\text { Risco de infecção relacionado } \\
\text { ao comprometimento da função } \\
\text { pulmonar e dos mecanismos de } \\
\text { defesa. }\end{array}$ & Controlar a infecção. & $\begin{array}{l}\text { Banho; Controle de doenças } \\
\text { transmissíveis; Controle de infecção; } \\
\text { Controle de imunização/vacinação; } \\
\text { Controle do ambiente; Cuidados com } \\
\text { lesões; Cuidado com sondas e } \\
\text { drenos; Supervisão da pele. }\end{array}$ \\
\hline
\end{tabular}

\title{
Considerações Finais
}

Com os dados apresentados, concluiu-se que a pneumonia nosocomial representa complicações frequentes, sendo um fator agravante para ocorrência de óbitos, com importante 111 
ISSN 2179-6750

relação com uso de ventilação mecânica invasiva, gerando, assim, elevado tempo de internação hospitalar e maior custo para o sistema de saúde.

Por essas razões, a profilaxia dessas infecções deve-se em grande parte à equipe multiprofissional que cuida do paciente, em especial a de enfermagem, que responde por vários mecanismos de prevenção, sejam em atividades de supervisão, administrativas, seja nos cuidados prestados aos pacientes admitidos nas enfermarias e UTI.

Sendo assim, é necessário dar ênfase a conscientização profissional, para buscar a excelência no atendimento com competência e habilidade profissional, objetivando a implantação e operacionalização de bundles da PAV, como meta prevenir a pneumonia.

\section{Referências}

1. Guyton AC; Hall JE. Tratado de Fisiologia Médica. 11 a ed. Rio de Janeiro, Elsevier Ed., 2006.

2. Chauvet P; Costata W; Faria AC. Pneumonia adquirida na comunidade. Revista Hospital Universitário Pedro Ernesto. Vol.9 N.2. 2010. [Acesso em 14/08/2014]. Disponível em: http://revista.hupe.uerj.br/detalhe_artigo.asp?id=158

3. Sociedade Brasileira de Pneumologia e Tisiologia. Diretrizes brasileiras para tratamento das pneumonias adquiridas no hospital e das associadas à ventilação mecânica. Jornal Brasileiro de Pneumologia. Vol.33 suppl. 1. São Paulo/SP, 2007. [Acesso em 10/10/2014]. Disponível em: http://www.scielo.br/scielo.php?pid=S180637132007000700001\&script=sci_arttext

4. Cervo AL, Berviam PA. Metodologia cientifica. 4.ed. São Paulo: Macron Books;1996.

5. Robbins SL; Vinay K e cols. Patologia Básica. Rio de Janeiro/RJ: Elsevier, p. 563, 2008.

6. Morais TMN, Silva A, Avi ALRO, Souza PHR, Knobel E, Camargo LFA. A importância da atuação odontológica em pacientes internados em unidade de terapia intensiva. Rev. bras. ter. intensiva [serial on the Internet]. 2006 Dec [cited 2015 Mar 04] ; 18(4): 412-417. Available from: http://www.scielo.br/scielo.php?script=sci_arttext\&pid=S0103507X2006000400016\&lng=en. http://dx.doi.org/10.1590/S0103-507X2006000400016.

7. Silva LCC. Condutas em pneumologia. Vol. 1 e 2. Rio de Janeiro - RJ: REVINTER, p. 388392; 942, 2001.

8. Ornelas CP; Cobucci RAS. Planos terapêuticos de enfermagem para o paciente com pneumonia. Revista Enfermagem Integrada. 2010; 3(1):395-407. 
ISSN 2179-6750

9. Gil AC. Como elaborar projetos de pesquisa. 3.ed. São Paulo: Atlas; 1999.

10. Junkes MB; Santos ML. Trabalhos acadêmicos: A facilidade em desenvolvê-los. Rolim de Moura/RO, 2007.

11. Porto CC. Semiologia Médica. 6. ed. Rio de Janeiro/RJ: Guanabara Koogan, p. 355-357, 2011.

12. Ganong LH. Integrative reviews of nursing research. Res Nurs Health. 1987; 10(1):1-11.

13. Dandagi GL. Nosocomial pneumonia in critically ill patients. Lung India. 2010; 3(27):149153.

14. Porth CM; Matfin G. Fisiopatologia. Vol. 2. Rio de Janeiro/RJ: Guanabara Koogan, p. 658, 2010.

15. Rodrigues JLN. Pneumonia Nosocomial (PN). Hospitais Universitários. 2010. [Acesso em 19/08/2014]. Disponível em: http://www.huwc.ufc.br/arquivos/biblioteca_cientifica /File/DIM/PROTOCOLOS\%20CLINICOS\%20HUWC/infectologia/pneumonianosocomial. pdf

16. Rufino R; Pugliese JG; Kirk K; Bartholo TP; Mafort TT. Pneumonia adquirida no hospital Visão Crítica. Revista Hospital Universitário Pedro Ernesto. Vol.9. N.2. 2010. [Acesso em 10/10/2014]. Disponível em: http://revista.hupe.uerj.br/detalhe_artigo.asp?id=159.

17. Ricci M. Incidência de pneumonia nosocomial em uma unidade de terapia intensiva geral de Cascavel - Paraná. Trabalho de Conclusão de Curso (Graduação em Fisioterapia). UNIOESTE. Cascavel/PR, 2005. 94p.

18. Freire ILS; Farias GM; Ramos CS. Prevenindo pneumonia nosocomial: cuidados da equipe de saúde ao paciente em ventilação mecânica invasiva. Revista Eletrônica. 2006; 8(3):37797.

19. Silveira IC et al. O Pulmão na prática médica. 4. ed. Rio de Janeiro: EPUB, p. 135, 1999.

20. Nanda International. Diagnóstico de Enfermagem da NANDA. Porto Alegre/RS: Artmed, 2014.

21. Smeltzer, Suzanne C.; BARE, Brenda G. Tratado de Enfermagem médico-cirúrgico. 10. ed. Rio de Janeiro: Guanabara Koogan, 2006.

22. Foschiera F; Viera CS. O diagnóstico de enfermagem no contexto das ações de enfermagem: percepção dos enfermeiros docentes e assistenciais. Revista Eletrônica de Enfermagem. 2004; 6(2):189-198. 
ISSN 2179-6750

23. Rezende CG. Estudo de caso de um paciente com pneumonia em Goiânia. Enfermagem Atual. Goiânia/GO. 2010. 20p.

24. Goldman L; Dennis CA. Tratado de Medicina interna. 23. ed. Rio de Janeiro/RJ: Elsevier, p. 2502, 2009.

25. Ranzani, OT; Prina E; Torres A. Nosocomial pneumonia in the intensive care unit: how should treatment failure be predicted? Revista Brasileira de Terapia Intensiva. 2014; 26(3):208-211.

26. Dochterman JM; Bulechek GM. Classificação das Intervenções de Enfermagem (NIC). 4. ed. Porto Alegre/RS: ARTMED, 2008.

27. Doenges $M$ et al. Planos de Cuidado de Enfermagem. 5. ed. Rio de Janeiro/RJ: Editora Guanabara Koogan, p. 141; 153,2003.

28. Johnson $M$ et al. Ligações entre NANDA, NOC E NIC: Diagnósticos, resultados e intervenções de enfermagem. 2.ed. Porto Alegre/RS: Artmed, 2009.

29. Sopena N, Heras E, Casas I, Bechini J, Guasch I, Pedro-Botet ML, Roure S, Sabrià M. Risk factors for hospital-acquired pneumonia outside the intensive care unit: A case-control study. American Journal of Infection Control. 2014: 42:38-42. 